\title{
Secondary food reinforcement versus exploratory drive in satiated rats'
}

ROCHELLE ABEND

UNIVERSITY OF CALIFORNIA, LOS ANGELES

A previous experiment, which found that satiated rats preferred a goal box containing food to an empty goal box, was replicated and modified to the extent necessary to examine the possibility of the presence of an exploratory drive. Four groups of 10 rats each ran down a runway, one trial per day for 20 days, to a goal box under one of four conditions: (1) primary reinforcement (accessible food), (2) secondary reinforcement (inaccessible food), (3) neutral reinforcement (inaccessible marbles), (4) no reinforcement (empty goal box). The group running to an empty goal box ran more slowly than the other three groups, whose running times were approximately equal. The increased speed of the groups with additional drive-irrelevant stimuli in the goal box indicates the possible operation of an exploratory drive.

In an attempt to demonstrate the learned value of food reward, Wike \& Casey (1954, Experiment II) found that satiated rats ran faster to a goal box with food than to one which was empty. Although they concluded that the sight of food served as an effective secondary incentive, they admitted the possible operation of an exploratory drive, in that food pellets might have served as objects to be explored, as learned rewards, or as a combination of the two. In this replication of their experiment, control groups for exploratory drive and accessibility of food effects were added. Method

The age, strain of Ss, apparatus, handling, training, running time measurement, and group matching procedures were identical to those used by Wike and Casey. After matching for running speed, 40 Ss were assigned randomly to four experimental conditions with $10 \mathrm{Ss}$ per condition. During the test series following training, Ss had food and water accessible at all time in their home cages.

The Ss received one test trial per day for 20 days. Then half the Ss in each condition received 10 massed test trials on day 21 , and the remaining half received 10 massed trials on day 22. In each condition, a $\mathrm{S}$ remained in the goal box for $20 \mathrm{sec}$. at the end of a trial. The content of the goal box defined the four conditions during the test series. Group $\mathbf{P}$ (primary reinforcement) ran to a goal box with food pellets strewn on the floor. Group S (secondary reinforcement) ran to mesh-covered food pellets. Group E exploratory reinforcement) ran to mesh-covered, multi-colored marbles. Group $N$ (no reinforcement) ran to an empty goal box.

\section{Results and Discussion}

Figure 1 gives the results of this replication along with those of the Wike and Casey experiment, in terms of median running times for each condition. The points (i), (ii) refer to the last two days of training. The remaining points are median running times for successive blocks of five trials. Trials 21-25 are the first five of the 10 massed trials, with Ss tested on days 21 and 22 grouped together; trials $26-30$ are the last five of the massed trials.

For the 20 spaced trials, an overall analysis of variance yielded a non-significant F-ratio among conditions. Comparing pairs of groups in blocks of five trials, no significant differences were found for the first two blocks. For blocks 11-15 and 16-20, the noreward group, group $\mathrm{N}$, ran significantly slower than each of the other groups, while the latter three did not differ in speed (Newman-Keuls test, Winer, 1962, $\mathrm{p}<.05)$.

Comparing medians as did Wike and Casey, the same order of results was obtained. An overall Wilcoxon test for $k$ samples (Walker \& Lev, 1953) showed no significant differences between groups. Individual comparisons for each block showed that group $N$ ran significantly slower $(p<.05)$ than groups $E$ and $P$ for trials 1-5; significantly slower than group $E$ for trials 6-10; and significantly slower than groups E, P, and $S$ for trials 11-15 and 16-20. Differences in all

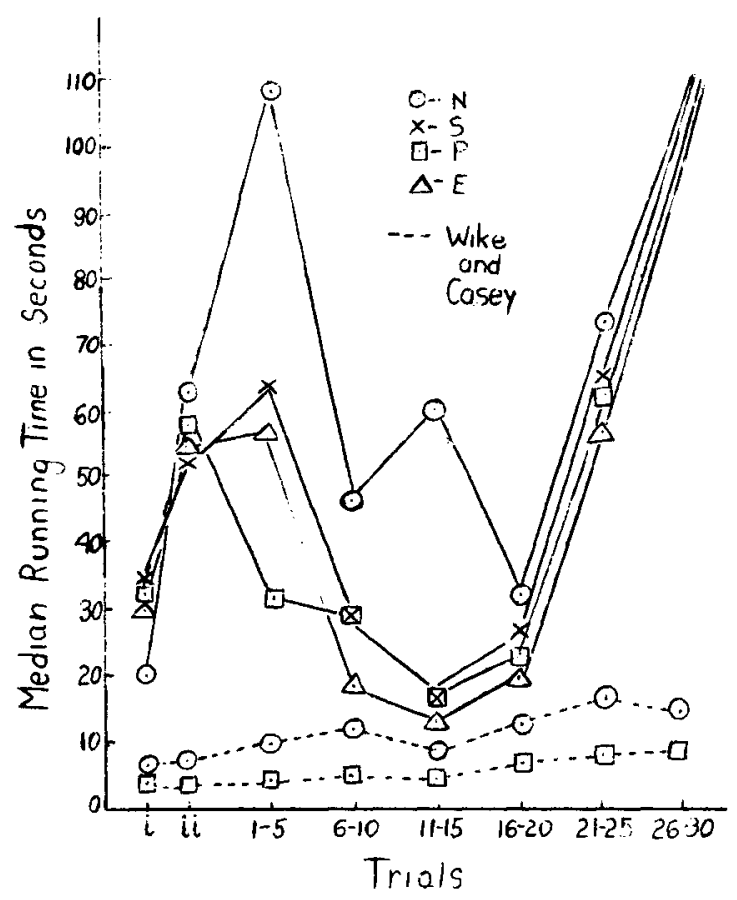

Fig. 1. 
pairwise comparisons on blocks among groups E, $P$, and $S$ were not significant. Similar analysis on the massed trials showed no significant differences although the relative order of groups $\mathrm{N}, \mathrm{P}, \mathrm{S}$, and $\mathrm{E}$ remained.

Thus, the results of Wike and Casey's experiment were replicated. Inspection of Fig. 1 indicates that their data were less variable and that their animals ran faster than those of the present study. However, the present results indicate the possible operation of an exploratory drive in the Wike and Casey study, since similar increases in running speed are observed for Ss running to marbles, accessible food, and inaccessible food, relative to those munning to an empty goal box.

The question of the presence of a secondary incentive is still open, for the results of this experiment could have been obtained if an exploratory drive were operative in group $E$ and a secondary incentive operative in groups $P$ and $S$. Since food was familiar, the exploratory drive might be weak relative to the learned drive in conditions $P$ and $S$. On the other hand, since the marbles were novel, they could elicit a stronger exploratory drive. In order to test the relative importance of secondary incentive and exploratory drive, an additional control group which had early experience with marbles as well as pellets would be needed.

\section{References}

Walker, H. M., \& Lev, J. Statistical inference. New York: Holt, Rinehart, \& Winston, 1953.

Wike, E. L., \& Casey, A. The secondary reward value of food for satiated animals. J. comp. physiol. Psychol., 1954, 47, 441-443. Winer, B. J. Statistical principles in experimental design. New York: McGraw Hill, 1962.

\section{Note}

1. I would like to acknowledge gratefully the help and supervision of Dr. T. Trabasso. 